\title{
Discrete Coupling and Synchronization in the Insulin Release in the Mathematical Model of the $\beta$ Cells
}

\author{
L. J. Ontañón-García ${ }^{1}$ and E. Campos-Cantón ${ }^{2}$ \\ ${ }^{1}$ Instituto de Investigación en Comunicación Óptica, Departamento de Físico Matemáticas, Universidad Autónoma de San Luis Potosí, \\ Álvaro Obregón 64, 78000 San Luis Potosí, SLP, Mexico \\ ${ }^{2}$ División de Matemáticas Aplicadas, Instituto Potosino de Investigación Científica y Tecnológica A.C., \\ Camino a la Presa San José 2055, Colonia Lomas 4a Sección, 78216 San Luis Potosí, SLP, Mexico
}

Correspondence should be addressed to L. J. Ontañón-García; luisjavier.ontanon@gmail.com

Received 19 October 2012; Revised 19 December 2012; Accepted 26 December 2012

Academic Editor: Gualberto Solís-Perales

Copyright ( 2013 L. J. Ontañón-García and E. Campos-Cantón. This is an open access article distributed under the Creative Commons Attribution License, which permits unrestricted use, distribution, and reproduction in any medium, provided the original work is properly cited.

\begin{abstract}
The synchronization phenomenon that occurs in the Langerhans islets among pancreatic $\beta$ cells is an interesting topic because these cells are responsible for the release of insulin in the blood stream. The aim of this work is to generate in-phase bursting electrical activity (BEA) in $\beta$ cells with different behaviors such as active, inactive, and continuous spiking cells based on mathematical models using a discrete time coupling. The approach considers two steps, the former is a mechanism on how to force $\beta$ cells to switch from silent phase to active, the latter is based on how to deal with in phase synchronization between active $\beta$ cells. The coupling signal is triggered in discrete events caused by the crossing of a threshold of an active $\beta$ cell which is given or defined by a Poincaré plane. The coupling on the inactive cells is applied to the state in which are the concentrations of agents which regulate the BEA. Based on numerical simulations, synchronization in the insulin release is obtained from $\beta$ cells with different behaviors.
\end{abstract}

\section{Introduction}

In nature, an interesting example of synchrony occurs in the coupling of pancreatic $\beta$ cells, which are responsible for insulin release in glucose homeostasis. The connected $\beta$ cells are in the islets of Langerhans as clusters among gap junction channels [1], and they exhibit a complex pattern of membrane-potential oscillations called BEA $[2,3]$. Recent results show that the cellular electrical activity during exocytosis occurs when a specific concentration of the agents regulating the protein release is reached (e.g., the calcium concentration in the endoplasmic reticulum [4]).

One of the principal characteristics among $\beta$ cells that is under investigation is that they produce BEA if they are not isolated from the cluster [5], causing electrical synchronization with its neighbors under certain considerations $[6,7]$.

The above commentary lays out a number of challenges in one specific area where mathematical models can help us address some of our biggest needs: how $\beta$ cells are coupled between them and synchronize in clusters. In the last decades, mathematical models have been designed in order to suffice the conditions met by the experimental data acquired by biologists. These models help to understand and reproduce specific behaviors such as the memory in the transmission delays in the neurons [8] and the bursting activity in most of the cells [9]. There are works focused on the research to understand the mechanisms by which the electrical activity appear, for example [10-15], the latest include the concentration vector of agents which regulate the BEA that have been discovered throughout the years, such as intracellular calcium, concentration of calcium and potassium in the endoplasmic reticulum, ADP (adenosine diphosphate), and glucose. With the aid of these models, some studies have been made in order to prove the synchronization among cells [16-19], the majority have focused on a coupling based on the electrical activity and the number of cells in the cluster.

However, if the electrical activity is a result of the internal process that occurs in the cell due to the permeation of some agents and it is known that not all cells burst in synchrony among the islet, then the coupling among cells must not only 
be given through electrical activity, but through the permeation and concentration of certain agents that regulate the insulin secretion in order that every inactive cell in the cluster begin to oscillate when the release of insulin is required, thus they synchronize with its neighbors. This work proposes a mechanism on how to force an inactive $\beta$ cell to an active state and synchronize in phase its electrical activity to the cluster of cells; to accomplish this, we use the detection of a threshold of the electrical activity of a regular active master cell via Poincaré planes as explained in [20]. The idea is to generate a driving signal which is triggered in discrete events caused by the crossing of a specific threshold by some master system with a previously defined Poincaré plane.

The paper is organized as follows: In Section 2, the mathematical model of the $\beta$ cell is described. Section 3, explains the forced coupling method via discrete events. In Section 4, we propose a master-slave coupling to produce in-phase BEA among $\beta$ cells with different behaviors (active, inactive, and periodic burst). Finally, conclusions are made about how this approach might impact coupling and synchrony among cells.

\section{Beta-Cell Model}

It is known that the mathematical models of the $\beta$ cell present two phases called active and silent phases. Each phase corresponds to a rapid and slow oscillation of the membrane potential, respectively. The active phase is related to the insulin-glucose response of the cells, and it has been proven that at lower concentrations of glucose, the intact cells in the islets do not burst, while at intermediate concentrations only a fraction burst [21].

The mathematical model implemented throughout the paper has been taken from Pernarowski [15]. Here the behavior of a single cell coupled in a cluster of cells was described. Using fast and slow variables, his model may describe also inactive cells. The model is given as follows:

$$
\begin{gathered}
\dot{u}=f(u)-\omega-c, \\
\dot{\omega}=\omega_{\infty}(u)-\omega, \\
\dot{c}=\epsilon(h(u)-c),
\end{gathered}
$$

where $u$ is the membrane potential, $\omega$ is a channel activation parameter for the voltage-gated potassium channel, and $c$ are concentrations of agents which regulate the BEA, such as intracellular calcium and concentration of calcium in the endoplasmic reticulum and ADP. form:

The functions $f(u), \omega_{\infty}(u)$ and $h(u)$ take the following

$$
\begin{gathered}
f(u)=\frac{-a}{3} u^{3}+a \widehat{u} u^{2}+\left(1-a\left(\widehat{u}-\eta^{2}\right)\right) u \\
\omega_{\infty}(u)=\left(1-\frac{-a}{3}\right) u^{3}+a \widehat{u} u^{2}-\left(2+a\left(\widehat{u}-\eta^{2}\right)\right) u-3, \\
h(u)=\beta\left(u-u_{\beta}\right),
\end{gathered}
$$

where the parameters are tuned to the next values for an active cell: $a=1 / 4, \eta=3 / 4, \widehat{u}=3 / 2, \beta=4, u_{\beta}=-0.954$, and $\epsilon=1 / 400$. Using this specific values, the system exhibits square-wave bursting which is analogous to the BEA in the pancreatic beta cell.

Figure 1 depicts the states of (1) in time using Runge Kutta with an integration step equal to 0.01 . We have considered throughout the paper $t$ as the number of iterations times the integration step; Figure 1(a) shows the membrane potential $u$ which is triggered due to the levels of glucose in the blood. Figure 1(b) shows the channel activation parameter for the voltage-gated potassium channel $\omega$, and Figure 1(c) shows the concentration of calcium $c$.

It can be seen that when the concentration of calcium $c$, due to the levels of glucose in the blood, increases, the membrane potential and the channel activation parameter for the voltage-gated potassium channel $\omega$ commences an active phase with square-wave bursting. This system presents only one equilibrium point $Q=(-0.6,-1.609,1.416)$, with corresponding eigenvalues $\lambda=(-1.946,0.978,0.002)$.

As Pernarowski has shown in [15], the system given by (1) is considered an inactive system by changing the slow variable $u_{\beta}=-2$; this is appreciated from Figures $1(\mathrm{~d}), 1(\mathrm{e})$, and $1(\mathrm{f})$, which shows the states of the system due to this change. The parameter $c$ shown in Figure 1(f) increases until it gets near 2, and the cell exhibits a stationary behavior rather than bursting. This is one of the main problems on nonfunctional $\beta$ cells.

Others refer to cells that present continuous spiking activity which is commonly attributed to isolated cells (see [18] and the reference within) and sometimes to cells belonging to clusters with a reduced number of cells in it [22]. The system given by (1) presents spiking activity by changing the fast parameters $\eta=1, \widehat{u}=3 / 2$. The states of this system may be appreciated in Figures 1(g), 1(h), and 1(i).

Based on these characteristic behaviors of $\beta$ cells, we propose a forced coupling in order to generate BEA in the inactive cell as is described in Section 3.

\section{Forced Coupling Based on Discrete Events}

The forced coupling is enabled by discrete time via Poincaré plane as described in [20], in which a master system is responsible for activating other inactive systems; that is, every time the master system crosses a threshold defined by a Poincaré plane then a forcing signal is activated in order to constrain a forced system. Since the coupling signal is generated each crossing event, the triggering is considered discrete in time. A brief description on how to yield this coupling is included in the appendix.

We are going to consider a master system as $\dot{\mathbf{x}}=$ $\left[\dot{u}_{m}, \dot{\omega}_{m}, \dot{c}_{m}\right]^{T}$, where $u_{m}, \omega_{m}, c_{m}$ correspond to the states of (1) with the parameters described above for an active $\beta$ cell. The Poincaré plane is located at $u=0$, it can be seen in Figure 2 with the gray dashed line. This location has been chosen in order to detect the membrane potential of the cell when the active phase begins. Figure 2 also depicts the $u$ state of the master $\beta$ cell. Each crossing event is marked with a red asterisk. 


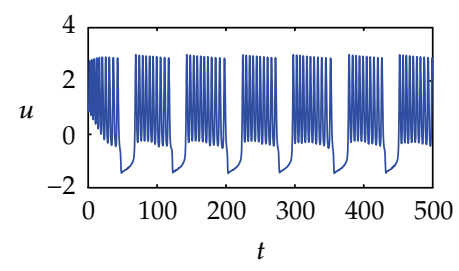

(a)

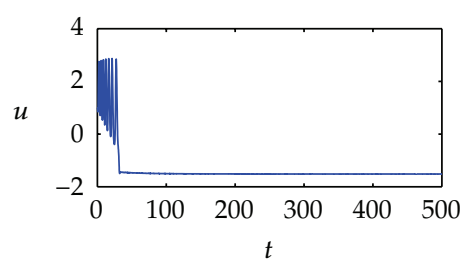

(d)

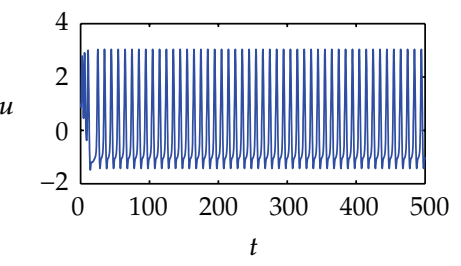

(g)

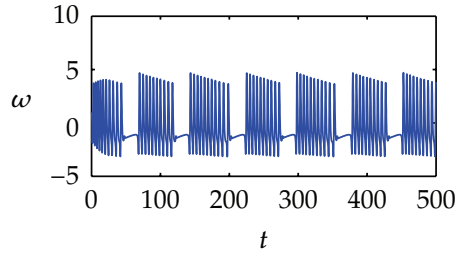

(b)

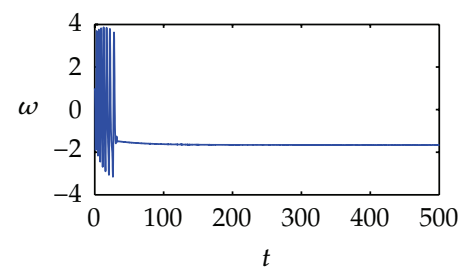

(e)

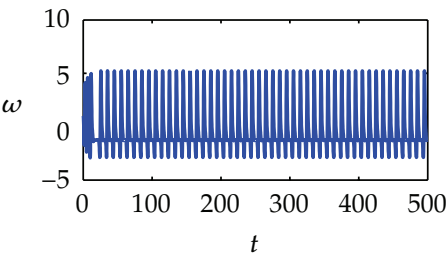

(h)

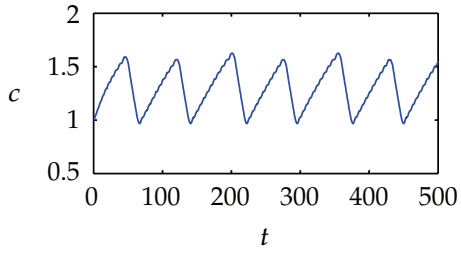

(c)

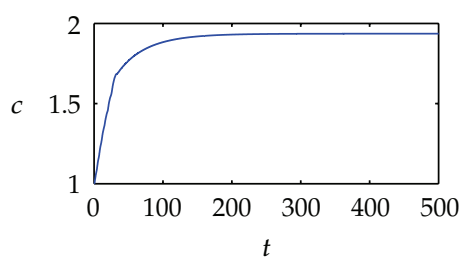

(f)

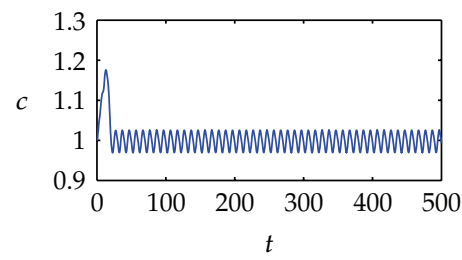

(i)

FIGURE 1: Time series of the system states given by (1) for an active cell in (a), (b), and (c) with $u_{\beta}=-0.954$; an inactive cell in (d), (e), and (f) with $u_{\beta}=-2$; continuous spiking (h), (i), and (j) with the fast parameters $\eta=1, \widehat{u}=3 / 2$ : in (a), (d), and (g), the membrane potential $u$; (b), (e), and (h) voltage gate potassium $\omega$; (c), (f), and (i) concentration of calcium $c$.

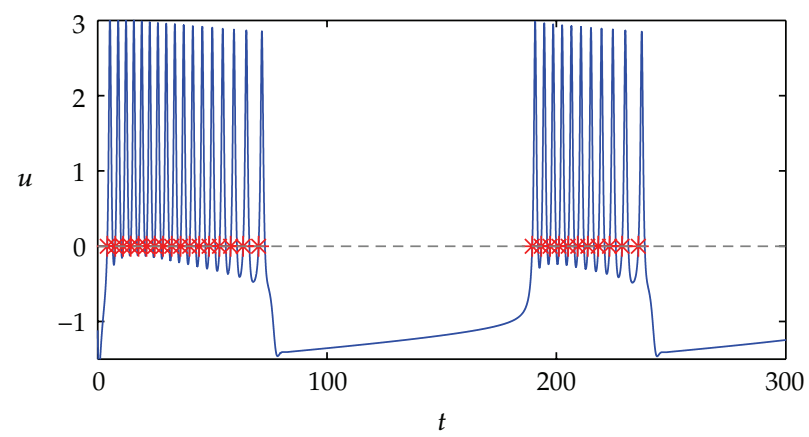

Figure 2: Time series of the $u$ state of an active $\beta$ cell as the master system with (1) for some iterations in time. The gray dashed line marks the Poincaré plane, and each crossing event is marked with red asterisks.

The forced system is given by the following equation:

$$
\dot{\mathbf{y}}=\left[\begin{array}{c}
\dot{u}_{s} \\
\dot{\omega}_{s} \\
\dot{c}_{s}
\end{array}\right]+\left[\begin{array}{l}
0 \\
0 \\
\xi
\end{array}\right] .
$$

This system is considered to be an inactive $\beta$ cell for the corresponding parameter $u_{\beta}=-2$ as described before. Where $\xi \in \mathbf{R}$ is the coupling signal. Here $\xi$ takes the form $\xi=A e^{-\tau\left(t-t_{i}\right)} \cos \left(w\left(t-t_{i}\right)\right)$ from (A.2).

First we adjust the coupling strength $A=-1$ and the coupling is activated in $t_{c}=500$. Figure 3 (a) shows the time series corresponding to the master system and the coupled system in red and blue line, respectively. Notice that both systems behave autonomously, and at the time $t_{c}$ the inactive system is coupled. The forced system starts to oscillate periodically out of phase of the master system, and for each two active phases of the master system, the forced system remains inactive one period of time, so we call this period of time as skipping one active phase. The reason for this skipping may be appreciated in Figure 3(b) where the time series of the $c$ state is depicted. It results that the coupled state does not reach the value of 1 as the master system does. By increasing the coupling strength $A=-1.3$, the skipping disappears as Figures $3(\mathrm{c})$ and $3(\mathrm{~d})$ show. 


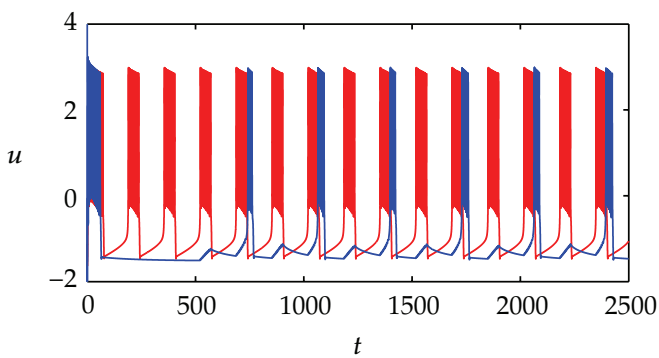

(a)

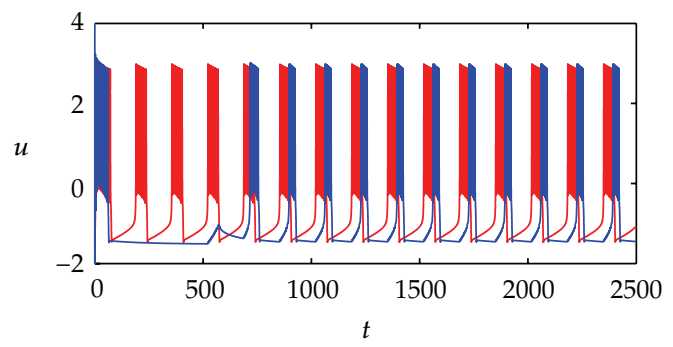

(c)

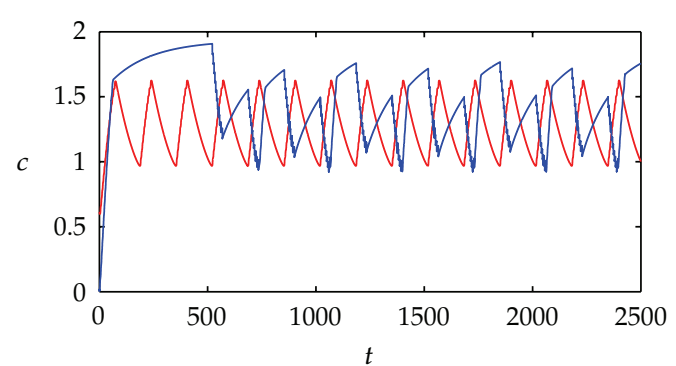

(b)

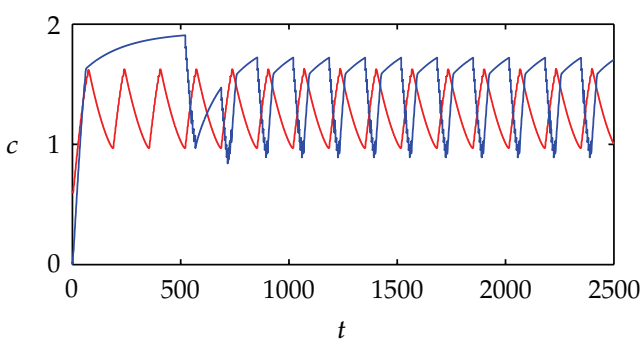

(d)

FIGURE 3: Time series of the system states $u$ and $c$ of the coupled inactive system from (3) marked with the blue line; the states $u$ and $c$ of the master system marked with the red line for a different coupling strength $A$. The coupling starts at $t_{c}=500$. For (a) and (b), $A=-1$. For (c) and (d), $A=-1.3$.

The proposed coupling can make an inactive $\beta$ cell to produce BEA. However, the bursts occur out of phase from the master system, this is because there is no interaction considered from the connection between the cells in gap junctions. The electrical activity in the membrane potential synchronizes the oscillations in the cells, so an additional coupling is proposed in Section 4 .

\section{In-Phase Oscillations through a Membrane Potential Coupling}

In order to force an inactive $\beta$ cell to produce BEA in phase with the master system, we propose the following unidirectional coupling:

$$
\dot{\mathbf{y}}=\left[\begin{array}{c}
\dot{u}_{s} \\
\dot{\omega}_{s} \\
\dot{c}_{s}
\end{array}\right]+\left[\begin{array}{c}
k\left(u_{m}-u_{s}\right) \\
0 \\
\xi
\end{array}\right]
$$

where $u_{m}$ represents the state of the master system, $u_{s}$ stands for a negative feedback of the inactive system, and $k \in \mathbf{R}$ stands for the strength of the unidirectional coupling. Setting this strength to $k=1$ and the starting time of the coupling $t_{c}=500$ and keeping the same value of $A=-1.3$, it results that the system from (4) becomes active and produces oscillations. These oscillations are now in phase with the master system; however, the amplitude obtained in the bursting differs significantly; this is shown in Figure 4(a). By incrementing the unidirectional coupling strength to $k=2$, this difference is diminished as it is appreciated in Figure 4(b), where the amplitudes are almost equal.
Now that the inactive cell has been coupled and forced to produce in-phase BEA with the master system, it is straightforward to think if this type of coupling works with the other (active or continuous spiking) $\beta$ cells belonging to the islet, because it would be unlikely to apply it only to the inactive cells.

So we considered two more systems to apply the coupling:

$\dot{\mathbf{v}}=\left[\begin{array}{c}\dot{u}_{s} \\ \dot{\omega}_{s} \\ \dot{c}_{s}\end{array}\right]+\left[\begin{array}{c}k\left(u_{m}-u_{s}\right) \\ 0 \\ \xi\end{array}\right] ; \quad \dot{\mathbf{z}}=\left[\begin{array}{c}\dot{u}_{s} \\ \dot{\omega}_{s} \\ \dot{c}_{s}\end{array}\right]+\left[\begin{array}{c}k\left(u_{m}-u_{s}\right) \\ 0 \\ \xi\end{array}\right]$,

where $\dot{\mathbf{v}}$ refers to an active $\beta$ cell with the same parameters as $\dot{\mathbf{x}}$. And $\dot{\mathbf{z}}$ refers to a continuous spiking $\beta$ cell with the fast parameters $\eta=1, \widehat{u}=3 / 2$. The coupling parameters are the same $A=-1.3, k=2$, and $t_{c}=500$. Figure $5(a)$ shows the active $\beta$ cell before $t_{c}$ produce BEA out of phase with the master system, when the system is coupled after $t_{c}$ begins to oscillate in-phase but with a reduced amplitude in the bursts.

A similar thing results when coupling a continuous spiking $\beta$ cell, before the coupling the system oscillate autonomous, after $t_{c}$ the coupled system oscillate in phase with the master system but with the same diminution in amplitude as in Figure 5(b).

Based on the above results, we conjecture that even if the coupling is applied to an islet with different behaviors in its containing cells, all cells are constrained to produce BEA in phase. As the BEA is related to the insulin secretion we can say that all cells are synchronized in the release of insulin. 


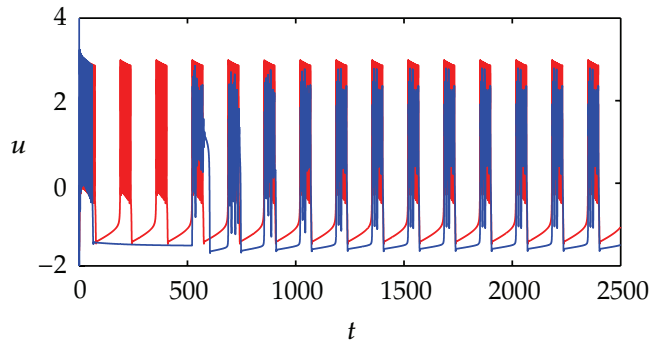

(a)

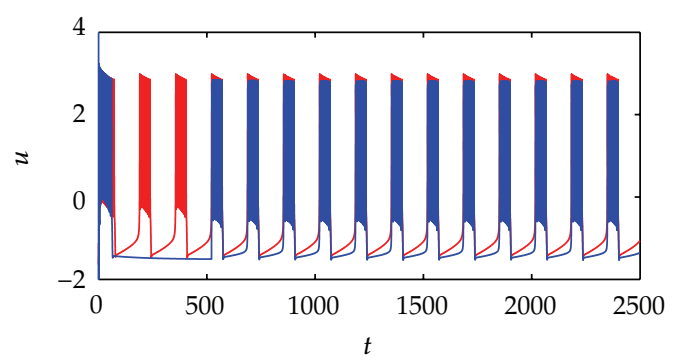

(b)

FIGURE 4: Time series of the system state $u$ of the coupled inactive (response or slave) system from (4) marked with the blue line; the state $u$ of the master system marked with the red line for a different unidirectional coupling strength $k$. The coupling starts at $t_{c}=500$ and $A=-1.3$. For (a) $k=1$ and (b) $k=2$.

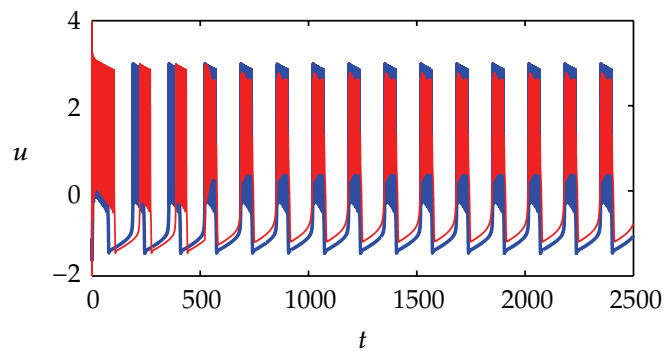

(a)

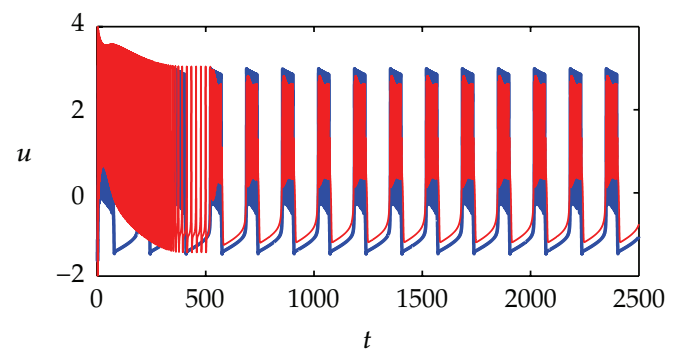

(b)

Figure 5: Time series of the system states $u$ of the coupled active and continuous spiking systems. Marked with the blue line the state $u$ of the master system given by (1), and marked with the red line the states of; (a) the active $\beta$ cell with $u_{\beta}=-0.954$. (b) the continuous spiking $\beta$ cell with the fast parameters $\eta=1, \widehat{u}=3 / 2$. The unidirectional coupling strength $k=2, t_{c}=500$ and $A=-1.3$.

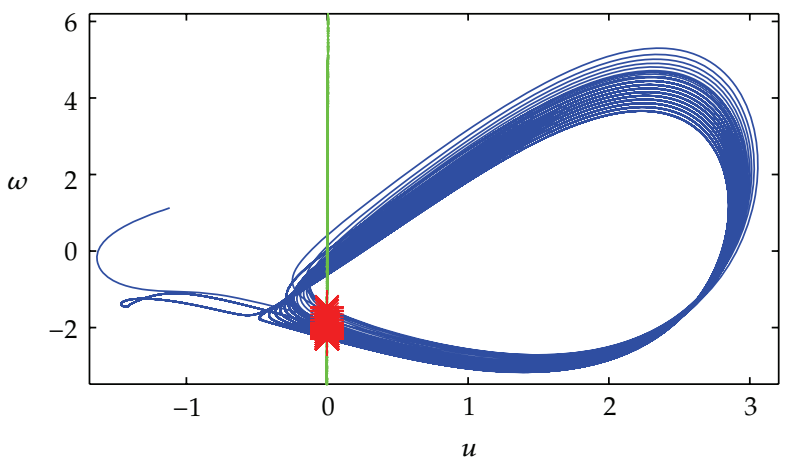

(a)

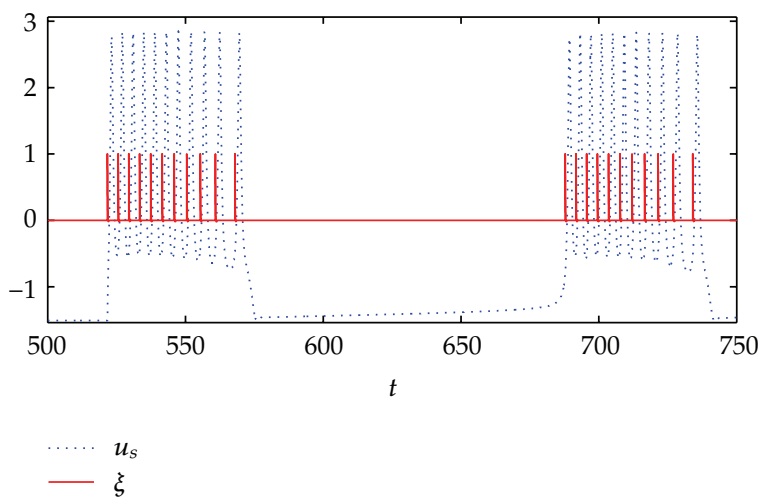

(b)

Figure 6: Projection of the master $\beta$-cell system onto the plane $(u, \omega)$ intersected by the Poincaré plane $\Sigma$. The points of each crossing event $\left\{\varphi_{m\left(\mathbf{x}_{0}\right)}^{t_{0}}, \varphi_{m\left(\mathbf{x}_{0}\right)}^{t_{1}}, \varphi_{m\left(\mathbf{x}_{0}\right)}^{t_{2}}, \ldots\right\}$ are marked with asterisk. (b) Signal $\xi(t)$ from a $\beta$-cell system marked in solid red line, and state $u_{1}$ of the autonomous system marked with dashed line.

\section{Conclusions}

The synchronization in the BEA among $\beta$-cells is a very important topic to consider because due to this phenomenon, the regulation of glucose is carried out. Several experiments have determined that under specific conditions a number of $\beta$ cells in the islet may produce different behaviors than the regular ones, causing the synchronization to disappear. Using a forced coupling method applied to a mathematical model of the cell, we demonstrate that inactive $\beta$ cells can be forced to produce out of phase BEA via the detection of a threshold in an active master $\beta$ cell. In order to synchronize in phase this activity, we applied a unidirectional coupling and demonstrate that even cells with different commonly 
behaviors such as active or continuous spiking can produce BEA in phase with the master cell synchronizing the active phases which are related to the release of insulin in the islets.

\section{Appendix}

\section{Activation of the Coupling Signal $\xi$}

Although the coupling signal $\xi$ can be generated in several ways, usually it is considered as a periodical signal; but in order to avoid the periodicity, we take the model of trigger given by [20]; here the generation of the coupling signal is briefly explained. Consider an autonomous system described as

$$
\mathbf{x}^{\prime}=F(\mathbf{x}), \quad F: \mathbf{R}^{m} \longrightarrow \mathbf{R}^{m}
$$

which is monitored by a Poincaré plane $\Sigma:=\left\{\left(\mathbf{x}_{1}, \mathbf{x}_{2}, \mathbf{x}_{3}\right)\right.$ : $\left.\alpha_{1} \mathbf{x}_{1}+\alpha_{2} \mathbf{x}_{2}+\alpha_{3} \mathbf{x}_{3}+\alpha_{4}=0\right\}$ where $\alpha_{1}, \ldots, \alpha_{4} \in \mathbf{R}$ are arbitrary coefficients of a hyperplane equation whose values are considered arbitrarily according to the following discussion. We are interested in the crossing events of the trajectory of the autonomous system equation (1) which generates the attractor $\mathscr{A}_{x}$. The Poincaré plane $\Sigma$ crosses the attractor $\mathscr{A}_{x}$, generating the points $\left\{\varphi_{m}^{t_{0}}\left(\mathbf{x}_{0}\right), \varphi_{m}^{t_{1}}\left(\mathbf{x}_{0}\right), \varphi_{m}^{t_{2}}\left(\mathbf{x}_{0}\right), \ldots\right\} \in \Sigma$ at each crossing event. Where $\varphi_{m}^{t_{i}}\left(\mathbf{x}_{0}\right)$ is the flow restricted to $\mathscr{A}_{x}$ for the initial condition $\mathbf{x}_{0}$. Therefore, we can specify the following time series $\Delta_{\mathbf{x}_{0}}=\left\{t_{0}, t_{1}, t_{2}, \ldots\right\}$, which depends on the initial conditions of the system in (1). The location of the plane must be located in order to meet the condition $\mathscr{A}_{x} \cap \Sigma \neq \emptyset$, assuming that at least one crossing event at time $t_{i}$ exists. Throughout this work we have focused on the crossing events of the trajectory of the master system with $\Sigma$ in only one direction. So the time series $\Delta_{\mathbf{x}_{0}}$ contains each crossing event that satisfies $(d / d t)\left(x_{1}\right)>0$. Following the above discussion, the term $\xi(t)$ from (1) is determined as follows:

$$
\xi(t)=\left(A e^{-\tau\left(t-t_{i}\right)} \cos \left(w\left(t-t_{i}\right)\right), 0,0\right)^{T}
$$

where $\tau \in \mathbf{R}$ represents an underdamping factor which allows us to modulate the signal and the scalar $w \in \mathbf{R}$ stands for the frequency. Therefore, the underdamped signal is triggered with each crossing event of (1) with $\Sigma$. Figure 6(a) shows the projection of an active $\beta$ cell system with the equations given by (1).

The autonomous system is monitored by a Poincaré plane with values $\alpha_{1}=1, \alpha_{2}=0, \alpha_{3}=0$, and $\alpha_{4}=0$; every event of crossing between the system and the plane $\Sigma$ is marked with an asterisk. And the form of the signal (A.2) generated is depicted in Figure 6(b).

\section{Acknowledgments}

L. J. Ontañón-García is a Doctoral Fellow of CONACYT at the Graduate Program on Applied Science at IICO-UASLP. E. Campos-Cantón acknowledges CONACYT for the financial support through Project no. 181002.

\section{References}

[1] P. Meda, A. Perrelet, and L. Orci, "Gap junctions and $\beta$-cell function," Hormone and Metabolic Research, vol. 10, Supplement 10 , Biochemistry and Biophysics of the pancreatic $\beta$-cell, pp. 157-161, 1980.

[2] P. M. Dean and E. K. Matthews, "Electrical activity in pancreatic islet cells," Nature, vol. 219, no. 5152, pp. 389-390, 1968.

[3] P. M. Dean and E. K. Matthews, "Glucose-induced electrical activity in pancreatic islet cells," Journal of Physiology, vol. 210, no. 2, pp. 255-264, 1970.

[4] C. Amatore, S. Arbault, I. Bonifas, M. Guille, F. Lemaître, and Y. Verchier, "Relationship between amperometric pre-spike feet and secretion granule composition in Chromaffin cells: an overview," Biophysical Chemistry, vol. 129, no. 2-3, pp. 181-189, 2007.

[5] P. Smolen, J. Rinzel, and A. Sherman, "Why pancreatic islets burst but single $\beta$ cells do not: the heterogeneity hypothesis," Biophysical Journal, vol. 64, no. 6, pp. 1668-1680, 1993.

[6] P. Meda, I. Atwater, and A. Goncalves, "The topography of electrical synchrony among $\beta$-cells in the mouse islet of Langerhans," Quarterly Journal of Experimental Physiology, vol. 69, no. 4, pp. 719-735, 1984.

[7] G. T. Eddlestone, A. Goncalves, J. A. Bangham, and E. Rojas, "Electrical coupling between cells in islets of langerhans from mouse," Journal of Membrane Biology, vol. 77, no. 1, pp. 1-14, 1984.

[8] X.-P. Yan and W.-T. Li, "Global existence of periodic solutions in a simplified four-neuron BAM neural network model with multiple delays," Discrete Dynamics in Nature and Society, vol. 2006, Article ID 57254, 18 pages, 2006.

[9] J. Duarte, L. Silva, and J. Sousa Ramos, "Computation of the topological entropy in chaotic biophysical bursting models for excitable cells," Discrete Dynamics in Nature and Society, vol. 2006, Article ID 60918, 18 pages, 2006.

[10] I. Atwater, C. M. Dawson, A. Scott, G. Eddlestone, and E. Rojas, "The nature of the oscillatory behaviour in electrical activity from pancreatic $\beta$-cell," Hormone and Metabolic Research, vol. 10, Biochemistry and Biophysics of the Pancreatic $\beta$-cell, pp. 100-107, 1980.

[11] T. R. Chay, "On the effect of the intracellular calcium-sensitive $\mathrm{K}+$ channel in the bursting pancreatic $\beta$-cell," Biophysical Journal, vol. 50, no. 5, pp. 765-777, 1986.

[12] D. M. Himmel and T. R. Chay, "Theoretical studies on the electrical activity of pancreatic $\beta$-cells as a function of glucose," Biophysical Journal, vol. 51, no. 1, pp. 89-107, 1987.

[13] T. R. Chay, "Effect of compartmentalized $\mathrm{Ca}^{+}$ions on electrical bursting activity of pancreatic $\beta$-cells," American Journal of Physiology, vol. 258, no. 5, pp. C955-C965, 1990.

[14] J. Keizer and P. Smolen, "Bursting electrical activity in pancreatic $\beta$ cells caused by $\mathrm{Ca}^{2+}$ and voltage-inactivated $\mathrm{Ca}^{2+}$ channels," Proceedings of the National Academy of Sciences of the United States of America, vol. 88, no. 9, pp. 3897-3901, 1991.

[15] M. Pernarowski, "Fast and slow subsystems for a continuum model of bursting activity in the pancreatic islet," SIAM Journal on Applied Mathematics, vol. 58, no. 5, pp. 1667-1687, 1998.

[16] A. Sherman and J. Rinzel, "Model for synchronization of pancreatic $\beta$-cells by gap junction coupling," Biophysical Journal, vol. 59, no. 3, pp. 547-559, 1991.

[17] C. L. Stokes and J. Rinzel, "Diffusion of extracellular $K^{+}$can synchronize bursting oscillations in a model islet of Langerhans," Biophysical Journal, vol. 65, no. 2, pp. 597-607, 1993. 
[18] G. De Vries and A. Sherman, "Channel sharing in pancreatic $\beta$-cells revisited: enhancement of emergent bursting by noise," Journal of Theoretical Biology, vol. 207, no. 4, pp. 513-530, 2000.

[19] J. M. W. Van De Weem, J. G. B. Ramírez, R. Femat, and H. Nijmeijer, "Conditions for synchronization and chaos in networks of $\beta$-cells," in Proceedings of the 2nd IFAC Conference on Analysis and Control of Chaotic Systems (CHAOS '09), pp. 176181, June 2009.

[20] L. J. Ontañón-Garcìa, E. Campos-Cantón, R. Femat, I. CamposCantón, and M. Bonilla-Marìn, "Multivalued synchronization by poincaré coupling," Communications in Nonlinear Science and Numerical Simulation. In press.

[21] P. M. Beigelman, B. Ribalet, and I. Atwater, "Electrical activity of mouse pancreatic $\beta$ cells. II. Effects of glucose and arginine," Journal de Physiologie, vol. 73, no. 2, pp. 201-217, 1977.

[22] A. Sherman, J. Rinzel, and J. Keizer, "Emergence of organized bursting in clusters of pancreatic $\beta$-cells by channel sharing," Biophysical Journal, vol. 54, no. 3, pp. 411-425, 1988. 


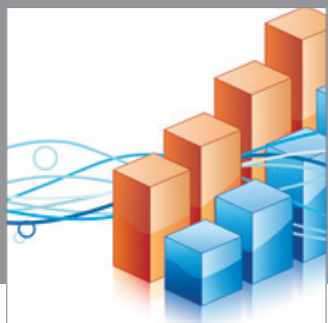

Advances in

Operations Research

mansans

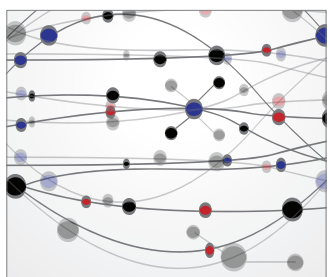

The Scientific World Journal
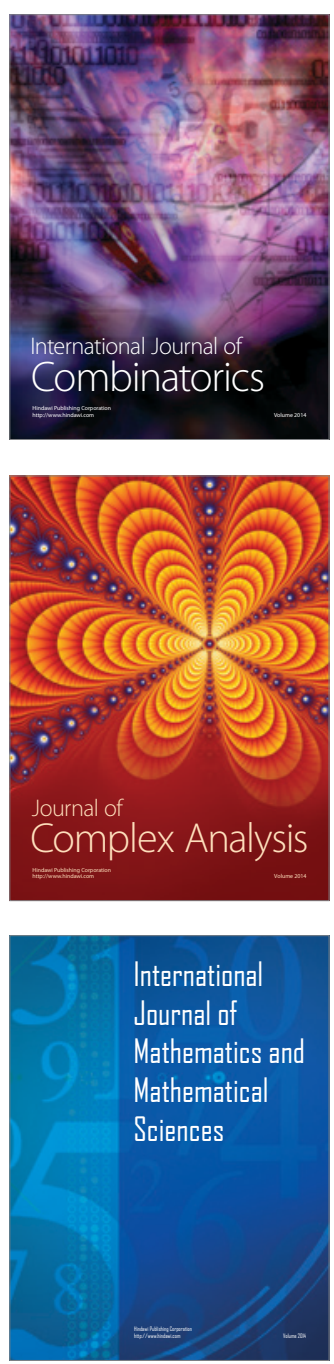
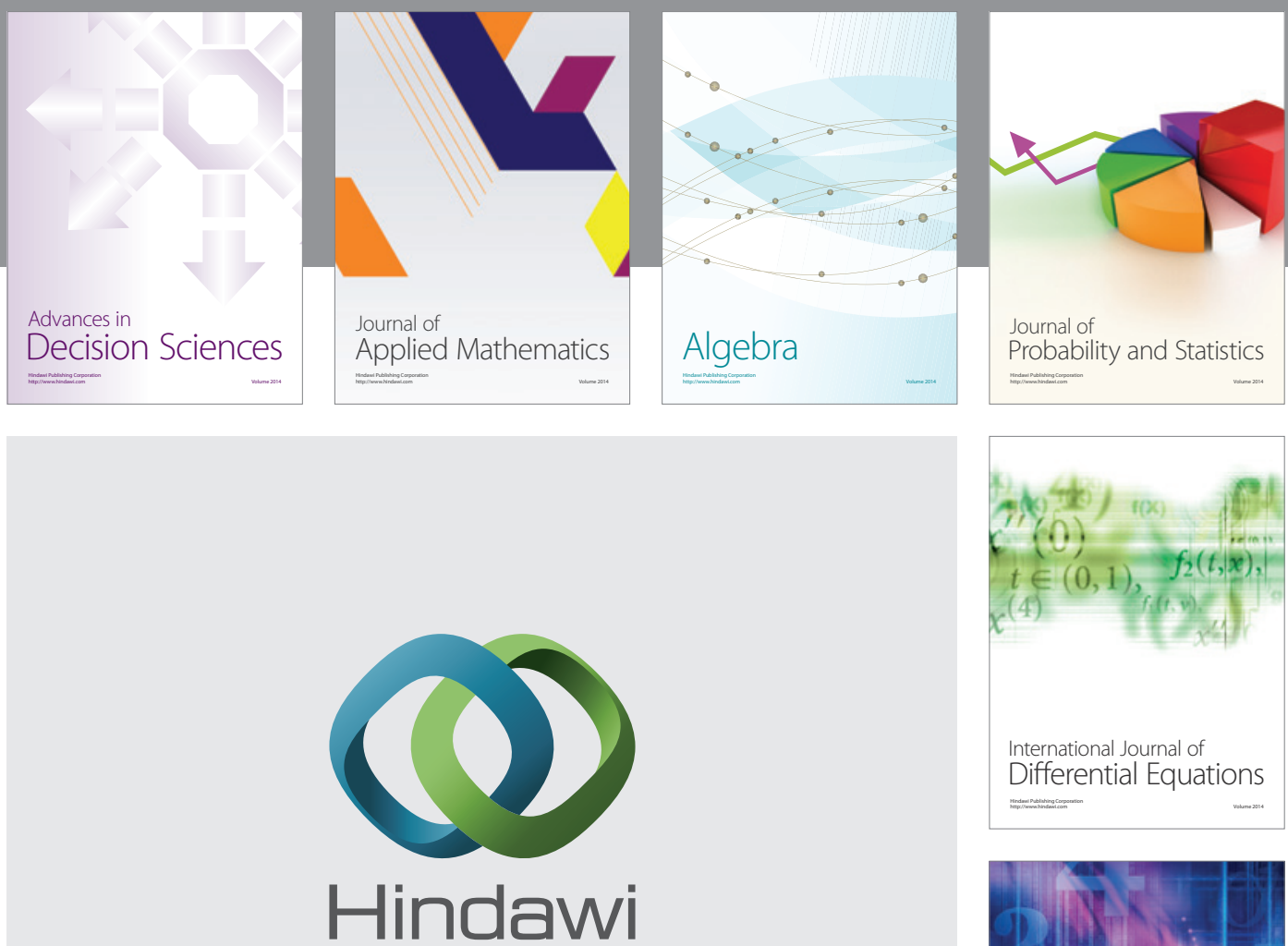

Submit your manuscripts at http://www.hindawi.com
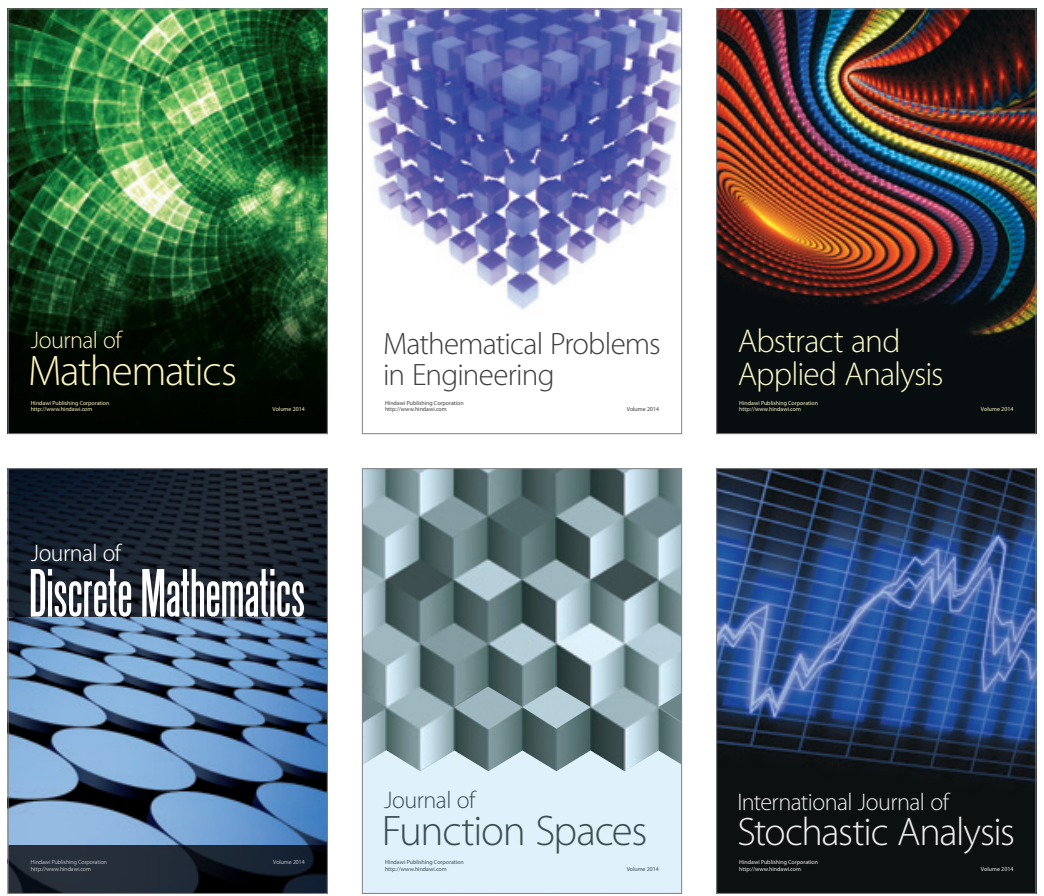

Journal of

Function Spaces

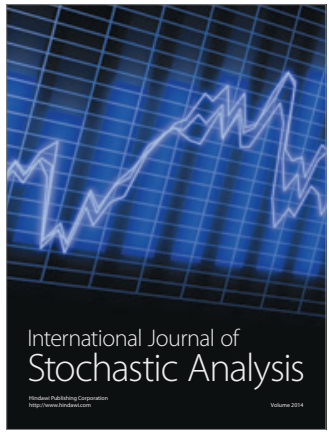

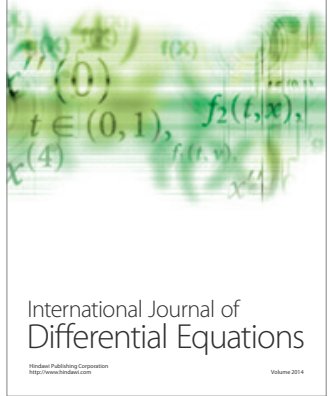
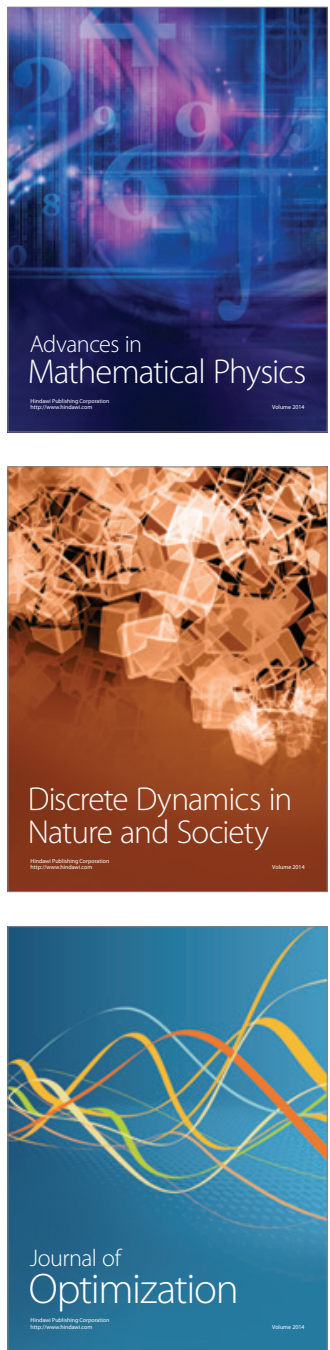